Bio - grafía. Escritos sobre la Biología y su Enseñanza. ISSN 2027-1034

Edición Extraordinaria. p.p. 1510 - 1515

Memorias del IX Encuentro Nacional de Experiencias en Enseñanza de la Biología y la Educación Ambiental. IV Congreso Nacional de Investigación en Enseñanza de la Biología.

\title{
PROMOVIENDO LA SUSTENT ABILIDAD AMBIENTAL A TRAVÉS DE UNA EXPERIENCIA CON EL USO DE PLANTAS TRADICIONALES EN EL TRAT AMIENTO DE PATOLOGÍAS INFLAMATORIAS.
}

\section{PROMOTING ENVIRONMENT AL SUSTAINABILITY THROUGH AN EXPERIENCE WITH THE USE OF TRADITIONAL PLANTS IN THE TREATMENT OF INFLAMMATORY PATHOLOGIES}

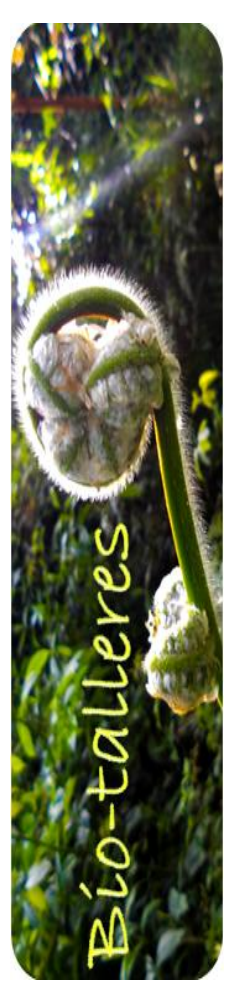

\author{
Paola Andrea Muñoz Castro' \\ Pilar Constanza Cely Castro ${ }^{1}$ \\ Daniel Felipe Rayo Alape ${ }^{1}$ \\ Julie Benavides Melo'
}

\section{RESUMEN}

Actualmente, en los tratamientos de patologías inflamatorias se evidencian propuestas tradicionales, ancestrales y farmacológicas para el control de vectores que originan el desbalance homeostático de los organismos, pretendiendo que con el uso de una única vía se llegue a una resolución satisfactoria. Desconociendo la influencia que otros factores ejercen sobre los seres humanos y que se suman complicando los cuadros de salud. Por ejemplo, asociando la predisposición a algunas enfermedades con estilos de vida nefastos y hábitos como la mala alimentación, sedentarismo, estrés, factores ambientales, etc.

Adicionalmente, la medicina tradicional ha dejado de lado la sabiduría ancestral que venía de la mano con abuelitos que conocían su territorio y tenían la capacidad de tomar del medio aquello que les permitiera controlar la afección. Por ello, en la actualidad, muchos de esos saberes ancestrales han quedado relegados y tratamientos que pueden ser complementarios, se abordan con una sola óptica. Consecuentemente, algunos métodos tradicionales han disminuido su efectividad y han acarreado una serie de efectos adversos producto de su implementación.

El propósito del taller es proponer un escenario donde se materialicen fundamentos del paradigma del buen vivir de modo que los saberes se encuentren y se complementen, generando un espacio de socialización y diálogo entre fundamentos ancestrales y bioquímicos dando mayor sustento a la utilización de plantas tradicionales para el tratamiento de patologías; además de invitar a la población en general a recurrir a la medicina natural y aprovechar todos los beneficios que esta puede ofrecer.

\footnotetext{
${ }^{1}$ Estudiante de Licenciatura en Química de la Universidad Pedagógica Nacional e investigador de SISMA "semillero de investigación en salud y medio ambiente".

${ }^{2}$ Docente de Licenciatura en Química de la Universidad Pedagógica Nacional, integrante del grupo ambiente y currículo y coordinadora de SISMA "semillero de investigación en salud y medio ambiente".
} 
Bio - grafía. Escritos sobre la Biología y su Enseñanza. ISSN 2027-1034

Edición Extraordinaria. p.p. 1510 - 1515

Memorias del IX Encuentro Nacional de Experiencias en Enseñanza de la Biología y la Educación Ambiental. IV Congreso Nacional de Investigación en Enseñanza de la Biología.

PALABRAS CLAVE: Plantas tradicionales, estrés oxidativo, especies oxidantes, patologías inflamatorias, buen vivir.

\begin{abstract}
Nowadays, traditional, ancestral and pharmacological proposals for the control of vectors that give rise to the homeostatic imbalance of organisms are evidenced in the treatment of inflammatory pathologies, claiming that with the use of a single route a satisfactory resolution is reached. Not knowing the influence that other factors exert on the human beings and that they add complicating the pictures of health. For example, associating predisposition to some diseases with harmful lifestyles and habits such as poor diet, sedentary lifestyle, stress, environmental factors, etc.
\end{abstract}

In addition, traditional medicine has put aside the ancestral wisdom that came hand in hand with grandparents who knew their territory and had the ability to take from the environment that would allow them to control the condition. For that reason, at present, many of these ancestral knowledges have been relegated and treatments that can be complementary, are approached with a single optics. Consequently, some traditional methods have diminished their effectiveness and have led to a series of adverse effects resulting from their implementation.

The purpose of the workshop is to propose a scenario where fundamentals of the paradigm of good living are materialized so that the knowledge is found and complemented, generating a space of socialization and dialogue between ancestral and biochemical foundations giving greater support to the use of traditional plants for Treatment of pathologies; In addition to inviting the population in general to resort to natural medicine and to take advantage of all the benefits that this can offer.

KEYWORDS: Traditional plants, oxidative stress, oxidizing species, inflammatory pathologies, good living.

\title{
INTRODUCCIÓN
}

"Actualmente la medicina tradicional es un recurso fundamental para la salud humana. Las plantas y árboles empleados son la base para el desarrollo de la medicina moderna, y en algunas zonas rurales e indígenas, son el único recurso del que disponen a falta de instituciones médicas y recursos monetarios para la adquisición de medicina moderna". (Escamilla \& Moreno, 2015)

Algunas plantas han sido empleadas por él hombre para curar enfermedades, desequilibrios físicos, mentales o sociales que se padecían desde tiempos inmemorables. Las plantas fueron la medicina original en todas las culturas y las grandes civilizaciones, desempeñando un rol fundamental (estas eran el medio para curar enfermedades en las personas), prácticas que fueron realizadas inicialmente de manera empírica; de este modo todos esos conocimientos fueron empleados en la formulación de diagnósticos y prevención de enfermedades, confiando exclusivamente en una experiencia práctica, experimental y a su vez su divulgación de generación en generación de forma oral o escrita. (Hernández \& López 2012) 
Bio - grafía. Escritos sobre la Biología y su Enseñanza. ISSN 2027-1034

Edición Extraordinaria. p.p. 1510 - 1515

Memorias del IX Encuentro Nacional de Experiencias en Enseñanza de la Biología y la Educación Ambiental. IV Congreso Nacional de Investigación en Enseñanza de la Biología.

Una planta medicinal de acuerdo con la OMS (1979), "es definida como cualquier especie vegetal que contiene sustancias que pueden ser empleadas para propósitos terapéuticos o cuyos principios activos pueden servir de precursores para la síntesis de nuevos fármacos; además de su importancia en la aplicación de la medicina moderna". (Hernández \& López. 2012)

La medicina tradicional ha sido muy significativa en la cultura de muchos pueblos, pues durante siglos fue el único recurso empleado para restablecer la salud de nuestros antepasados, por lo anterior tiene sentido ver a la medicina tradicional o natural como una fuente de alternativas para el tratamiento de patologías que por tanto contribuye a mejorar la calidad de vida restaurando nuestra salud y además facilita seguir una filosofía basada en la visión de los pueblos y nacionalidades indígenas "el buen vivir", como una oportunidad para construir una sociedad sustentada en la convivencia del ser humano en diversidad y armonía con la naturaleza, a partir del reconocimiento de los diversos valores culturales existentes en cada país y en el mundo. (Acosta, A. \& Gudynas, E. 2011). El intelectual Aymara David Choquehuanca, sostiene que el vivir bien es "recuperar la vivencia de nuestros pueblos, recuperar la cultura de la vida y recuperar nuestra vida en completa armonía y respeto mutuo con la madre naturaleza, con la Pachamama, donde todo es vida, donde todos somos uywas, criados de la naturaleza y del cosmos". (Gudynas, 2011)

En la actualidad son muchas las patologías que aquejan a la población mundial, dichas patologías según investigaciones tienen sus inicios en los famosos radicales libres los cuales son átomos o grupos de átomos que se caracterizan principalmente por tener un electrón desapareado es decir, desde un punto de vista químico como aquellas especies las cuales se encuentran cargadas o no, presentando de esta manera en su estructura atómica un electrón desapareado o impar en el orbital externo, dándole una configuración espacial que genera gran inestabilidad. (Venéreo, G. 2002)

Los radicales libres son elementos fundamentales en el metabolismo, pero también constituyen un riesgo, especialmente para las células y las biomoléculas, como los ácidos nucleicos, las proteínas, polisacáridos y lípidos cuando superan en número a mecanismos antioxidantes internos. (Ceballos, Guapillo, Jiménez, Maldonado \& Méndez, 2010).

En condiciones fisiológicas normales, nuestro organismo, neutraliza los radicales libres a través de varios mecanismos antioxidantes, con el fin de prevenir el daño oxidante. "Cuando la capacidad de control de las sustancias oxidantes es superada, se establece una situación conocida como estrés oxidante". El cual llega a relacionarse con numerosas enfermedades tales como: Cáncer, diabetes y alteraciones vasculares. (Ceballos et al. 2010)

Una de las patologías que se presumen es generada por acción de radicales libres es el cáncer. "El cáncer es un conjunto de enfermedades de origen multifactorial. Se caracteriza principalmente por un crecimiento anormal, una proliferación acelerada y no controlada de las células, con capacidad de formar un tumor y metástasis en diferentes tejidos. El estrés oxidante y el proceso tumoral se encuentran estrechamente relacionados a través de la oxidación del material genético". (Ceballos et al. 2010) 
Bio - grafía. Escritos sobre la Biología y su Enseñanza. ISSN 2027-1034

Edición Extraordinaria. p.p. 1510 - 1515

Memorias del IX Encuentro Nacional de Experiencias en Enseñanza de la Biología y la Educación Ambiental. IV Congreso Nacional de Investigación en Enseñanza de la Biología.

Otras de esas patologías son la cardiopatía isquémica y el infarto agudo del miocardio. Patologías que ponen de manifiesto procesos que comienzan con exceso de radicales libres, iniciando el evento aterosclerótico cuando un radical libre sustrae en el lumen vascular un electrón a la grasa poli-insaturada del colesterol LDL (lipoproteína de baja densidad); éste se oxida y da inicio a la formación de la placa ateroesclerótica y la disfunción del endotelio vascular que permite el paso del LDL oxidado al espacio subendotelial. (Ceballos et al. 2010)

Por último, en consonancia con el PDI-UPN en su eje 4. Universidad y Sustentabilidad Ambiental y específicamente con relación al proyecto de inversión que se encuentra en ejecución código 410.705.4.1.2, denominado "Fortalecimiento de la participación de la UPN en redes ambientales nacionales e internacionales" al cual se articula la presente propuesta. Se considera que es importante mencionar que este tipo de abordajes que ponen en diálogo conocimientos ancestrales con explicaciones emitidas desde la ciencia, se constituyen como escenarios propicios para el fomento del pensamiento complejo.

Así, estas iniciativas pueden cristalizar esfuerzos desde diferentes áreas del conocimiento para trabajar en torno a la promoción de prácticas de sustentabilidad ambiental que interesen a diversos grupos poblacionales y sobre todo que logren gestar trabajo colaborativo entre instituciones, grupos o semilleros de investigación, profesionales, estudiantes, entre otros actores; redundando en trabajo en red para garantizar la concreción de rutas y metas mucho más integrales.

\section{PROPÓSTOS}

- Socializar mediante una estrategia didáctica la articulación entre fundamentos ancestrales y bioquímicos para la utilización de plantas tradicionales en el tratamiento de patologías.

- Acopiar y valorar los conocimientos de la medicina ancestral que han sido transmitidos de generación en generación.

- Explorar otra(s) alternativa(s) que puedan complementar o reemplazar la medicina convencional, proporcionando los beneficios esperados mientras se minimizan costos y efectos secundarios.

- Establecer mediante la información recogida dentro del taller, la posibilidad de generar trabajo colaborativo con otras instituciones que estén interesadas en promover temas de sustentabilidad ambiental y en lo posible proyectar una ruta para concretar acciones de trabajo.

- Obtener insumos para elaborar una ruta pedagógica que permita la alfabetización científica en las temáticas a trabajar o temas de interés que pueda ser aplicada en la Universidad Pedagógica Nacional o en cualquier institución que pueda requerirlo.

- Reconocer y promover el trabajo en redes, visibilizando individuos, semilleros, grupos de investigación, etc que tengan afinidad con el trabajo en temas afines a nuestra propuesta. 
Bio - grafía. Escritos sobre la Biología y su Enseñanza. ISSN 2027-1034

Edición Extraordinaria. p.p. 1510 - 1515

Memorias del IX Encuentro Nacional de Experiencias en Enseñanza de la Biología y la Educación Ambiental. IV Congreso Nacional de Investigación en Enseñanza de la Biología.

\section{ASPECTOS PROCEDIMENT ALES}

Metodología.

- El taller se desarrollará en un ambiente que simula un supermercado (o una tienda, eso dependerá de los recursos físicos y logísticos con que se cuente). Dentro de él habrá una recepción, unos pasillos (o secciones) de comidas y lo que sería una figura de caja registradora. Y puede ser trabajado en un tiempo máximo de una hora.

- Cada una de las ubicaciones descritas dará paso a una parte de la estrategia pedagógica que se está implementando, dando lugar a tres partes.

- Durante la primera parte y en la recepción del supermercado, se realizará una conferencia corta (máximo 20 minutos) que contiene material conceptual sobre:

$$
\begin{aligned}
& \rightarrow \text { Salud } \\
& \rightarrow \text { Homeostasis } \\
& \rightarrow \text { Estrés oxidativo }
\end{aligned}
$$

$\rightarrow$ Radicales libres

$\rightarrow$ Antioxidantes

$\rightarrow$ Patologías inflamatorias

- En la segunda parte se dará paso a las secciones de comida. Al principio, cada participante del taller recibirá un "carrito" para llevar las cosas que quiere comprar y se le pedirá que adquiera sus artículos para el mercado. En cada una de las secciones se entregará información como sigue:

$\rightarrow$ Vídeos recopilatorios de plantas medicinales.

$\rightarrow$ Mensajes de un abuelito indígena en donde él nos comparte algunos de sus conocimientos.

$\rightarrow$ Paradigma del buen vivir.

$\rightarrow$ Comparación de contenidos nutricionales de frutas versus jugos de sobre, jugos de caja y etc.

$\rightarrow$ Pruebas para establecer la forma correcta de cómo se deben realizar jugos, infusiones y otras preparaciones; de manera que se garantice el aprovechamiento de todos los beneficios que las plantas proporcionan.

$\rightarrow$ Patologías inflamatorias; causas y consecuencias.

- En la tercera parte se pasará a la caja, en ese lugar cada participante hará el pago correspondiente por su compra (cumpliendo con parámetros de material didáctico) y 
Bio - grafía. Escritos sobre la Biología y su Enseñanza. ISSN 2027-1034

Edición Extraordinaria. p.p. 1510 - 1515

Memorias del IX Encuentro Nacional de Experiencias en Enseñanza de la Biología y la Educación Ambiental. IV Congreso Nacional de Investigación en Enseñanza de la Biología.

el cajero le hará un balance de los costos asociados a hábitos de vida sana y/o vida saludable.

- Finalmente, en la salida habrá un buzón de sugerencias en el que el participante puede contar sus impresiones frente al taller y/o dejar sus datos para continuar en contacto con el grupo y establecer nuevas propuestas o profundizar en la que ha sido presentada.

\section{Materiales}

Tijeras, marcadores de colores, pegamento, cintas de colores, cinta pegante, cartón paja en octavos, cartulina, hojas blancas, lápices, computador y sala con Video Beam.

\section{BIBLIOGRAFÍA}

-Acosta, A. \& Gudynas, E. (2011). El buen vivir o la disolución de la idea del progreso recuperado de: https://goo.gl/ATYyVp

- Ceballos, G., Guapillo, M., Jiménez, E., Maldonado, O. \& Méndez, E. (2010). Radicales libres y su papel en las enfermedades crónico-degenerativas recuperado de: https://goo.gl//r2Yozo

- Hernández, D. \& López, S. (2012). Plantas medicinales recuperado de: https://goo.gl/ZG1kn9

- Organización Mundial de la Salud (2002) Estrategia de la OMS sobre medicina tradicional $2002-2005$.

- Gudynas, E. (2011). Buen vivir: Germinando alternativas al desarrollo recuperado de: https://goo.gl/LXAQSy

- Venéreo, G . J. (2002). Daño oxidativo, radicales libres y antioxidantes. Revista Cubana Medicina Militar. 2002;31(2):126-33. Instituto Superior de Medicina Militar "Dr. Luis Díaz Soto" 